\section{P52 PRISMS: A SYSTEMATIC REVIEW OF THE MRC 'PHASE IV' EVIDENCE ON IMPLEMENTING ASTHMA SELF- MANAGEMENT}

${ }^{1} \mathrm{H}$ Pinnock, ${ }^{2} \mathrm{E}$ Epiphaniou, ${ }^{3} \mathrm{HL}$ Parke, ${ }^{4} \mathrm{G}$ Pearce, ${ }^{2} \mathrm{SJC}$ Taylor. ${ }^{1}$ Asthma UK Centre for Applied Research, The University of Edinburgh, Edinburgh, UK; ${ }^{2}$ Multidisciplinary Evidence Synthesis Hub (MEsh), Queen Mary University of London, London, UK; ${ }^{3}$ Kings College London, London, UK; ${ }^{4}$ University of Coventry, Coventry, UK

\subsection{6/thoraxinl-2014-206260.193}

Aim Asthma self-management is effective in clinical trials, widely recommended by guidelines but poorly implemented. We aimed to synthesise the evidence from implementation studies of asthma self-management support interventions to inform delivery in routine clinical practice.

Methods Using systematic review methodology (Cochrane Handbook) we searched eight electronic databases, performed snowball and manual searches, and searched research databases for unpublished and on-going work. We included studies with a range of methodologies, and which evaluated the introduction of an asthma self-management support intervention in routine clinical practice. We assessed included papers for quality (Downs and Black), extracted and synthesised data on process (e.g. number of action plans issued) and clinical outcomes (e.g. measures of asthma control, unscheduled healthcare). Narrative synthesis used the whole systems approach as a framework. [PROSPERO registration: CRD42012002898].

Results We included 18 studies (7 randomised trials, 8 longitudinal database studies, 3 uncontrolled studies) from primary, secondary, community and managed care settings in six countries. Interventions which explicitly addressed patient, professional and organisational factors ( $\mathrm{n}=7$ studies) showed the most consistent improvement in both process and clinical outcomes. Targeting professionals ( $\mathrm{n}=2$ studies) improved process but not clinical outcomes. Targeting patients ( $n=6$ studies) had inconsistent impact on process/clinical outcomes. Targeting the organisation ( $\mathrm{n}=3$ studies) improved process, and had a small effect on clinical outcomes. Authors highlighted the importance of a healthcare system committed to supporting self-management, skills training for professionals, patient education programmes supported by regular reviews, and on-going evaluation of the implementation process.

Conclusion Effective interventions were complex: actively engaging patients, and training and motivating professionals within the context of an organisation which prioritised supported self-management. Commissioners and providers of services for people with asthma should consider how they can promote a culture of supporting self-management as a normal, expected, monitored and remunerated aspect of the provision of care.

Funding NIHR HS\&DR programme (project number 11/1014/ 04). The views and opinions expressed therein are those of the authors and do not necessarily reflect those of the Hs and DR programme, NIHR, NHS or the Department of Health.

\section{P53 MANCHESTER DESERT ISLAND QUESTION}

${ }^{1} \mathrm{KE}$ George, ${ }^{1} \mathrm{HGT}$ Brice, ${ }^{1} \mathrm{SJ}$ Fowler, ${ }^{2} \mathrm{U}$ Holmes, ${ }^{2} \mathrm{R}$ Daly, ${ }^{2} \mathrm{RM}$ Niven. ${ }^{1}$ University of Manchester, Manchester, Greater Manchester; ${ }^{2}$ University Hospital South Manchester, Manchester, Greater Manchester

\subsection{6/thoraxjnl-2014-206260.194}

Objectives To determine if specific clinical questions could reliably predict the ultimate inflammatory phenotype of patients

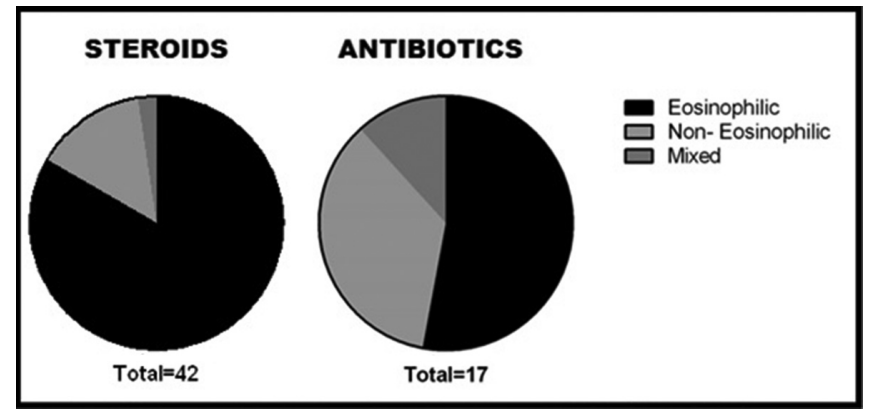

Abstract P53 Figure 1 Pie charts to show the different phenotypes relating to the answer of the desert island question

attending a severe asthma service. The severe asthma clinic at University Hospital of South Manchester now routinely ask the desert island question (DIQ).... "If you were on a desert island and could only have steroids or antibiotics which would you choose?"

Background It is widely accepted that asthma is the result of complex pathological processes giving rise to a variety of different phenotypes, including eosinophilic and non- eosinophilic inflammatory patterns. Phenotype is determined by investigations e.g. baseline blood/sputum eosinophil level and clinical response to treatments including steroid augmentation/withdrawal. Investigations include antibody status to Haemophilus Influenza and Streptococcus Pneumonia.

Methods New patients referred to the severe asthma service were asked the DIQ during their first appointment, prior to any investigations. We collected data from first clinic letters and case notes. Data collected included DIQ answer, phenotype, additional diagnoses, historical steroid and antibiotic use, sputum data and all asthma treatments trialled.

Results A total of 59 patients were identified. 71\% answered steroids and $29 \%$ answered antibiotics. Of those that answered steroids, $83 \%$ had eosinophilic asthma, 14\% had non-eosinophilic asthma. The breakdown of the different phenotypes within the different answer groups is illustrated in Figure 1. The positive predictive value (PPV) of the answer "steroids" was $86 \%$ and "antibiotics" $47 \%\left(\chi^{2}=7.182 \mathrm{p}=<0.01\right) .67 \%$ of the "antibiotic" answers and $38 \%$ of the "steroid" answers were found to be antibody deficient (Haemophilus Influenza and/or Streptococcus Pneumonia). 30\% who answered "antibiotics" were on maintenance steroids compared to $41 \%$ of patients who answered "steroids".

Conclusion This small pragmatic study has shown that the answer "steroids" to the DIQ has a high PPV for eosinophilic airway inflammation. The "antibiotic" response is much less useful in predicting inflammatory phenotype, but it does identify a high likelihood of antibody deficiency.

\section{P54 WHICH SECONDARY CARE ASTHMA PATIENTS ARE MOST LIKELY TO OVERESTIMATE THEIR CONTROL? A CROSS-SECTIONAL STUDY}

${ }^{1} \mathrm{~L}$ Patel, ${ }^{2} \mathrm{~J}$ Blakey, ${ }^{2} \mathrm{~K}$ Mortimer. 'University Hospital South Manchester, Manchester, UK; ${ }^{2}$ University Hospital Aintree, Liverpool, UK

\subsection{6/thoraxjnl-2014-206260.195}

Introduction Asthma is a global health issue affecting 300 million people worldwide; with increasing prevalence and morbidity and preventable mortality. Despite advances in management the number of asthma related deaths has not improved. 
Asthma is a heterogeneous condition, with a variety of clusters of clinical presentations and courses, objective measures and treatment responses. A common feature of asthma is the underreporting of poor symptom control by patients and under-recognition by clinicians. Poor asthma control in the preceding 12months prior to admission has been linked to asthma related deaths. The significance of measuring asthma control independently from asthma severity has been demonstrated. However, considerable differences in perceived and actual control are apparent. There is a need to identify patient groups at risk of under-reporting symptoms and not recognising poor control.

Aim To establish which patient features are associated with overestimation of disease control.

Setting Secondary care consultant led asthma clinic.

Population 108 patients recruited over 10 weeks.

Measures Objective measures of disease severity were mapped against perceived symptom control using the Asthma Control Test; age, gender, co-morbidities, medications, induced sputum, lung function, IgE, blood eosinophil, histamine challenge test, exhaled nitric oxide, ECG CXR, smoking status and BMI.

Analysis Significant associations between patient groups and perceptions of symptom control are described.

Results $61(56.6 \%)$ of patients had difficult asthma according to BTS guidance. 95 (88.0\%) had poorly controlled asthma, with $70(64.8 \%)$ of these perceiving adequate control of symptoms.

All patients with good perceived and actual control of symptoms; 13 (12.0\%), had never smoked. $85.5 \%$ of patients who did not recognise their symptoms prevalence were overweight, obese or morbidly obese.

All patients with raised IgE or blood eosinophillia had poorly controlled asthma; though $58.6 \%$ of this group perceived good control.

Conclusion This single centre cross-sectional study suggests smokers, overweight patients and those with inflammation predominant asthma are most likely to under-report severity. These findings are in keeping with the cluster analyses of Haldar and Moore. Further work is required to follow-up these patients to establish if poor perception of symptoms changes over time, or is associated with future asthma attack frequency.

\section{\begin{tabular}{|l|l}
\hline P55 IS PRESCRIPTION UPTAKE AND MEDICATION \\
\hline
\end{tabular} ADHERENCE RATING SCALE (MARS) A USEFUL TOOL IN ASSESSING ASTHMA CONTROL IN CHILDREN WITH PROBLEMATIC SEVERE ASTHMA (PSA)?}

${ }^{1} \mathrm{P}$ Nagakumar, ${ }^{1} \mathrm{P}$ Hall, ${ }^{1} \mathrm{M}$ Bracken, ${ }^{2} \mathrm{~S}$ Saglani, ${ }^{2} \mathrm{~A}$ Bush, ${ }^{2} \mathrm{~L}$ Fleming. ${ }^{1}$ Royal Brompton Hospital, London, UK; ${ }^{2}$ National Heart and Lung Institute, Imperial College, London, UK

\subsection{6/thoraxjnl-2014-206260.196}

Introduction Sub-optimal adherence to medications results in poor asthma control, however objective measurement of adherence is challenging.

Aim To assess adherence to inhaled corticosteroids (ICS) in children with problematic severe asthma (PSA) using prescription uptake and the Medicines Adherence Rating Scale (MARS) (selfreported adherence) and relate them to measures of asthma control.

Methods 160 patients assessed as part of the established $\mathrm{RBH}$ difficult asthma protocol (2008-2013) were included [[Arch Dis Child 2009;94:780-4]. Adherence was assessed using prescription uptake data (GP and local hospital) and MARS. Sub-optimal adherence was defined as a prescription uptake of $<80 \%$. Spirometry $\left(\mathrm{FEV}_{1}\right.$ pre bronchodilator), bronchodilator reversibility
Abstract P55 Table 1 Comparison of prescription uptake and measures of asthma control

\begin{tabular}{llll}
\hline & $\begin{array}{l}>0 \% \text { prescription } \\
\text { uptake }\end{array}$ & $\begin{array}{l}<0 \% \text { prescription } \\
\text { uptake }\end{array}$ & p value \\
\hline $\mathrm{FEV}_{1}(\%)$ & 81.5 & 78.5 & 0.38 \\
$\mathrm{BDR}^{2} \%$ & 5.3 & 8.7 & 0.81 \\
$\mathrm{FeNO}(\mathrm{ppb})$ & 22.5 & 28.8 & 0.20 \\
$\mathrm{ACT}^{* *}$ & 15 & 13 & 0.14 \\
MARS $^{*}$ & 24 & 22 & 0.10 \\
PAQLQ & 3.2 & 4.4 & 0.23 \\
Oral steroids in past 12 months & 7 & 7.5 & 0.80 \\
Hospital admissions in past 12 months & 1 & 2 & 0.98 \\
\hline
\end{tabular}

Data presented as median. Mann-Whitney test used to compare the groups. P value $<0.05$ is taken as significant. ${ }^{*}$ Max score 25 with higher scores indicating better self-reportedadherence. ${ }^{* *}$ Max score 25 , with higher scores indicating better asthma control.

(BDR), and exhaled nitric oxide (FENO) were measured. The Asthma Control Test (ACT), Paediatric Asthma Quality of Life Questionnaire (PAQLQ) was used to evaluate control. Number of courses of oral steroids and hospital admissions in the previous 12 months were recorded.

Results Median age was 11.6 yr (5-16). 66\% were male. 52\% had prescription uptake of $<80 \%$.

MARS score showed only a weak correlation with prescription uptake $\left(\mathrm{n}=48, \mathrm{r}^{2}=0.03, \mathrm{p}=0.29\right)$, even in patients with prescription uptake of $<50 \%\left(\mathrm{n}=23, \mathrm{r}^{2}=0.09, \mathrm{p}=0.32\right)$. No relationship was found between prescription uptake and ACT, MARS, FEV 1 , rescue courses of OCS, FeNO or BDR (Table 1).

Conclusion Poor prescription uptake was not related to any measure of asthma control, meaning that we could not differentiate the genuine therapy resistant from the non-adherent. But it is not possible to assess how much ICS was actually inhaled. Patterns of ICS use may be a more important determinant of control. Self-reported adherence, as measured by MARS was high even in those with very poor $<50 \%$ ) prescription uptake highlighting the limitations of this questionnaire. More objective means of assessing adherence should be incorporated into protocols for assessing severe asthma.

\section{P56 IMPACT OF PHARMACIST-LED ASTHMA AND COPD REVIEWS IN GENERAL PRACTICE}

H Khachi. Barts Health NHS Trust, London, UK

10.1136/thoraxjnl-2014-206260.197

Introduction Asthma and chronic obstructive pulmonary disease (COPD) account for a significant burden of disease in the UK. In a local initiative, a respiratory pharmacist carried out structured asthma and COPD reviews aimed at improving clinical outcomes in a primary care setting.

Aims and objectives This study aims to assess the impact of asthma and COPD reviews in accordance with national guidelines and standards of care.

Methods The study was carried out prospectively, during one day a week basis, over a twelve month period across six GP surgeries. Patients were included based on use of high dose inhaled corticosteroid and bronchodilator preparations (ICS/LABA) and/ or presence of previous exacerbations, accident and emergency (A\&E) or hospital admissions.

Results 231 patients with asthma $(\mathrm{n}=146,63.2 \%)$ and COPD ( $\mathrm{n}=85,36.8 \%)$ were reviewed, with 370 consultations carried 